\title{
Characterization of in vitro and in vivo metabolism of AG-024322, a novel cyclin-dependent kinase (CDK) inhibitor
}

\author{
Wei-Zhu Zhong ${ }^{*}$, Bojan Lalovic ${ }^{1}$, Jenny Zhan ${ }^{2}$ \\ ${ }^{1}$ Clinical Pharmacology, Pfizer Global Research and Development, New London, Connecticut, USA \\ ${ }^{2}$ Metabolism and Pharmacokinetics, Novartis Institute of Biomedical Research, Cambridge, Massachusetts, USA \\ *Pharmacokinetics, Dynamics and Metabolism, Pfizer Global Research and Development, San Diego, California, USA; \\ wei-zhu.zhong@pfizer.com
}

Received 16 November 2009; revised 25 November 2009; accepted 28 November 2009.

\begin{abstract}
Uncontrolled cell proliferation is the hall mark of many cancers, and is typically manifested by a deregulation of the cell-division cycle. CDKs play critical roles in regulating cell cycle, apoptosis and cell differentiation. AG-024322 is a multitargeted CDK inhibitor that has been shown to induce cancer cell apoptosis and demonstrate significant antitumor activity in human tumor xenograft models. This compound is under clinical development as an intravenous anticancer agent. AG-024322 exhibited moderate to high systemic clearance across preclinical species. In vitro metabolism in human liver microsomes and hepatocytes demonstrates that glucuronidation and oxidation represent the major metabolic pathways of AG-024322. The experiments of chemical inhibition and microsomes containing individual CYP or UGT isoforms revealed that CYP3A and UGT1A1 appear to predominantly mediate AG-024322 oxidation and glucuronidation, respectively. Formation kinetics of the two pathways in human liver microsomes suggested that the glucuronidation activity of AG-024322 was approximately 3-fold higher as compared to CYP-mediated oxidation, contributing approximately $37 \%$ and $13 \%$ of the total clearance, respectively, based on the projected human clearance. UGT1A1 is a polymorphic isoform involved in glucuronidation of bilirubin. It is of concern if glucuronidation via UGT1A1 plays a major role in the elimination of AG-024322 in humans as competitive inhibition of UGT1A1 has been associated with toxicity (Gilbert and Crigler-Najjar syndromes). Therefore, this information was used to influence the clinical study design to only include subjects having constitutive expression of UGT1A1 in
\end{abstract}

the first human study, thereby decreasing the potential risk of toxicity to patients.

Keywords: Microsomes; Hepatocytes; Reaction Phenotyping; Metabolite Formation Kinetics; CYP3A4; UGT1A1; Mass Balance in Rat

\section{INTRODUCTION}

AG-024322, $N$-(\{5-[3-(4,6-difluoro-1 $H$-benzimidazol-2yl)-1H-indazol-5-yl]-4-methylpyridin-3-yl $\}$ methyl)ethanamine, is a multitargeted CDK inhibitor under development as an anticancer agent. AG-024322 is a potent ATP-competitive inhibitor of the cell cycle kinases CDK1, CDK2, and CDK4, with kinetic inhibition constants (Ki) in the 1-3 nM range [1]. CDKs play a key role in regulating the cell cycle progression and to a large degree control cellular transitions from growth phases into phases associated with DNA replication and mitosis by phosphorylating several cellular proteins [2-4]. AG-024322 inhibits phosphorylation of CDK substrates in cells, resulting in cell arrest and broad-spectrum anti-proliferative activity, and has been shown to induce cancer cell apoptosis and significant antitumor efficacy in human tumor xenograft models with tumor growth inhibition (TGI) ranging from $32 \%$ to $86.4 \%$ [5].

Single-dose pharmacokinetics of AG-024322 was evaluated in the rat, dog and monkey following a 30 minute IV infusion, the intended administration route in humans. AG-024322 demonstrated moderate clearance of 26 and $27 \mathrm{ml} / \mathrm{min} / \mathrm{kg}$ in monkeys and dogs, respectively, and somewhat higher clearance in rats (67 $\mathrm{ml} / \mathrm{min} / \mathrm{kg}$ ), with half-lives ranging from 5 to 7 hours. Minimal contribution of renal clearance was observed in all 3 species $(\leq 1 \%)$. The volume of distribution greatly exceeded the volume of total body water across species (9 to $15 \mathrm{l} / \mathrm{kg}$ ), and was consistent with rapidly and 
widely tissue distribution observed in Long Evans rats by quantitative whole body autoradiography (QWBA) using $\left[{ }^{14} \mathrm{C}\right] \mathrm{AG}-024322$. The plasma protein binding of AG-024322 was in the range of 85 to $95 \%$ across species. In view of the predominant metabolic pathway of glucuronidation observed in human hepatocytes and the generally low metabolic turnover in in vitro incubations, the prediction of pharmacokinetics in human was performed using interspecies allometric scaling of singledose animal pharmacokinetics data. This approach lead to a reasonable prediction of human clearance $(12.5 \mathrm{ml} /$ $\mathrm{min} / \mathrm{kg}$ ), which was similar to the clearance actually observed in humans with IV doses from 20 to $340 \mathrm{mg}$. Detailed nonclinical pharmacokinetics and the prediction of human pharmacokinetics of AG-024322 will be reported elsewhere.

To support the development of AG-024322, a series of nonclinical in vitro and in vivo studies were conducted to identify AG-024322 metabolites, and to characterize the disposition of AG-024322. These studies involved with either using animal and human liver microsomes and hepatocytes incubated with C-14 labeled AG-024322 ( $\left.\left[{ }^{14} \mathrm{C}\right] \mathrm{AG}-024322\right)$ or using urine, plasma, bile, and fecal samples from rats administered $\left[{ }^{14} \mathrm{C}\right] \mathrm{AG}-024322$. Further studies to assess the two principal pathways of AG024322 biotransformation, CYP-mediated oxidative and UGT-mediated glucuronidation, and to identify the major enzymes involved in these metabolic pathways were also carried out.

In the present paper, discussion was emphasized on the in vitro and in vivo characterization of AG-024322 metabolism and the relative importance of CYP3A4 and UGT1A1 in the biotransformation of AG-024322 in HLM, as well as the relative contributions of the two metabolic pathways in the overall clearance of AG-024322.

\section{MATERIALS AND METHODS}

\subsection{Materials}

AG-024322 was synthesized at Pfizer Global Research \& Development (PGRD), La Jolla, CA. $\left[{ }^{14} \mathrm{C}\right] \mathrm{AG}-$ 024322 (Specific activity $53.1 \mathrm{mCi} / \mathrm{mmol}$ ) was synthesized by the Radiosynthesis Group at PGRD (Groton, $\mathrm{CT}$ ), and the label was located in the $\mathrm{C}-1$ position of the benzimidazol ring (Scheme 1). Rat, dog, monkey and human liver microsomes were obtained from Gentest Corporation. (Woburn, MA). Human liver microsome preparation (HL-Mix 14, $21.7 \mathrm{mg}$ protein/ml, $0.3 \mathrm{nmol}$ $\mathrm{CYP} / \mathrm{mg}$ protein) and microsomes from insect cells stably expressing human cDNA CYPs and UGTs (supersomes) were also purchased from Gentest Corporation. Cryopreserved hepatocytes for dogs, monkeys and humans, and the thawing media were obtained from In Vitro Technologies (Baltimore, MD). Sprague-Dawley rat hepatocytes and Hepatocyte Isolation Kit were pur-<smiles>CCNCc1cncc(-c2ccc3[nH]nc(-c4nc5c(F)cc(F)cc5[nH]4)c3c2)c1C</smiles>

Scheme 1. Chemical structure of AG-024322 (asterisk indicates the site of radiolabel).

chased from XenoTech LLC (Lenexa, KS). Ketoconazole, quindine, quercetin, furafyline, dibasic and monobasic potassium phosphate, NADPH, UDPGA, and WEM were purchased from Sigma-Aldrich Co. (St. Louis, MO). HEPES (4-(2-hydroxyethyl)-1-piperazineethanesulfonic acid) buffer was purchased from Invitrogen (Carlsbad, CA). Commercially obtained chemicals and solvents were of HPLC or the highest grade available.

\subsection{Liver Microsomal Incubations}

Liver microsomes were preincubated with AG-024322 and $\left[{ }^{14} \mathrm{C}\right] \mathrm{AG}-024322$ in $100 \mathrm{mM}$ potassium phosphate buffer ( $\mathrm{pH} 7.4$ ) for 5 minutes in a $37^{\circ} \mathrm{C}$ shaking water bath. The reaction was initiated by the addition of NADPH with a final incubation volume of $0.5 \mathrm{ml}$ containing $10 \mu \mathrm{M}$ AG-024322, $0.87 \mu \mathrm{M}\left[{ }^{14} \mathrm{C}\right] \mathrm{AG}-024322,1$ mM NADPH, $0.75 \mathrm{mg} / \mathrm{ml}$ microsomal protein and 100 $\mathrm{mM}$ potassium phosphate buffer $(\mathrm{pH} 7.4)$. The incubation was terminated at $0,10,20,40$ and 60 minutes by the addition of $0.5 \mathrm{ml}$ of acetonitrile and immediately vortexed and centrifuged at $1900 \times \mathrm{g}$ for 25 minutes. The supernatants were transferred to a fresh tube, evaporated to dryness under nitrogen, and reconstituted with $200 \mu \mathrm{l}$ of Solvent A for LC/RAM/MS analysis as described below.

\subsection{Hepatocytes Incubation}

Cryopreserved hepatocytes were thawed and used according to the manufacturer's instructions. The thawed cryopreserved hepatocytes were re-suspended into $\mathrm{CO}_{2}-$ bubbled incubation media to determine the total cell count using the Trypan Blue Exclusion method. The incubation media consisted of WEM supplemented with $10 \mathrm{mM}$ HEPES buffer. The final cell concentrations were $1.5,1.8,1$, and $1.23 \times 10^{6}$ viable cells $/ \mathrm{ml}$ for rat, $\mathrm{dog}$, monkey and human hepatocytes in WEM, respectively. Hepatocytes were incubated in the media containing 10 $\mu \mathrm{M}$ AG-024322 and $0.87 \mu \mathrm{M}\left[{ }^{14} \mathrm{C}\right] \mathrm{AG}-024322$ in a $\mathrm{CO}_{2}: \mathrm{O}_{2}(5: 95)$ stationary incubator at $37^{\circ} \mathrm{C}$. The final incubation volume was $1 \mathrm{ml}$. Reactions were terminated at several time points from time zero to 5 hours by the addition of $2 \mathrm{ml}$ of acetonitrile. Samples were then soni- 
cated, vortexed and centrifuged at $1900 \times \mathrm{g}$ for $25 \mathrm{~min}$ utes at $4^{\circ} \mathrm{C}$. The resulting supernatant was evaporated under nitrogen and reconstituted in $100 \mu \mathrm{l}$ of water for LC/RAM/MS analysis.

\subsection{CYP Reaction Phenotyping Screen}

AG-024322 (1 and $10 \mu \mathrm{M})$, each in triplicate, were incubated with a panel of microsomes from insect cells expressing individual human CYPs (Supersomes) containing 50 pmol of CYP1A2, 2C8, 2D6, 2E1, 3A4 and $3 \mathrm{~A} 5$ protein or $100 \mathrm{pmol}$ of $2 \mathrm{C} 9 * 1$ and $2 \mathrm{C} 19$. The final incubation volume was $0.5 \mathrm{ml}$. Incubations containing AG-024322 and $1 \mathrm{mM}$ NADPH in $100 \mathrm{mM}$ phosphate buffer ( $\mathrm{pH} 7.4$ ) were preincubated for 5 minutes at $37^{\circ} \mathrm{C}$. The reactions were started with the addition of $50 \mu \mathrm{l}$ of each CYP and were allowed to proceed up to 15 minutes. Incubations were stopped with the addition of $0.5 \mathrm{ml}$ of acetonitrile and $20 \mu \mathrm{l}$ of $1 \mathrm{ng} / \mu \mathrm{l}$ AG-024397 (IS), and immediately capped, vortexed and centrifuged at $1900 \times \mathrm{g}$ for 25 minutes at $4^{\circ} \mathrm{C}$. The supernatant of each sample was dried under $\mathrm{N}_{2}$ and reconstituted with $100 \mu \mathrm{L}$ of Solvent C for LC-MS analysis as described below.

\subsection{Chemical Inhibition}

CYP-specific chemical inhibitors, ketoconazole (CYP 3A4), quinidine (CYP2D6), quercetin (CYP2C8), and furafyline (CYP1A2) were used to identify the isoforms mediating the formation of N-desethyl AG-024322. Incubation mixtures were prepared with ketoconazole and quinidine at 20,100,500 and $1 \mu \mathrm{M}$, quercetin at 0.3 , 0.75 and $2 \mu \mathrm{M}$ and furafyline at 1,10 and $30 \mu \mathrm{M}$, and were preincubated with AG-024322 (1 and $10 \mu \mathrm{M})$ and $15 \mathrm{mg}$ HLM protein $(0.3 \mathrm{mg} / \mathrm{ml})$ for 5 minutes at $37^{\circ} \mathrm{C}$. Incubations were started by the addition of NADPH to a concentration of $1 \mathrm{mM}$ in a final volume of $0.5 \mathrm{ml}$. Incubations with furafyline, a time-dependent inhibitor of CYP1A2, required a preincubation step in the presence of $1 \mathrm{mM}$ NADPH for 20 minutes prior to the introduction of the substrate. The reactions were terminated after 8 minutes by the addition of $0.5 \mathrm{ml}$ of acetonitrile and 20 $\mu \mathrm{l}$ of $1 \mathrm{ng} / \mu \mathrm{l}$ IS. The remaining part of the procedure was performed as described above for CYP reaction phenotyping screen.

\subsection{UGT Reaction Phenotyping Screen}

AG-024322 (1 and $10 \mu \mathrm{M})$, each in triplicate, was incubated with 8 UGT isoforms (UGTlA1, 1A3, 1A4, 1A6, 1A8, 1A10, 2B7 and 2B15). The incubation procedure was adapted from Fisher et al. [6]. Microsomes $(0.5 \mathrm{mg}$ / $\mathrm{ml})$ suspended in $100 \mathrm{mM}$ phosphate buffer $(\mathrm{pH} 7.1)$ were initially treated with alamethicin at $0.025 \mathrm{mg} / \mathrm{mg}$ microsomal protein. The mixture also included $1 \mathrm{mM}$ $\mathrm{MgCl}_{2}$ and was kept on ice for 15 minutes to permealize the microsomal membrane. AG-024322 was then added and the mixture was preincubated at $37^{\circ} \mathrm{C}$ for 5 minutes.
The reaction was started with the addition of $1 \mathrm{mM}$ UDPGA in a total incubation volume of $0.2 \mathrm{ml}$, and were terminated after 15 minutes by the addition of $0.5 \mathrm{ml}$ of acetonitrile and $20 \mu \mathrm{l}$ of $1 \mathrm{ng} / \mu \mathrm{l}$ IS. The remaining part of the procedure was performed as described above for CYP reaction phenotyping screen.

\subsection{Metabolite Formation Kinetics in HLM}

The formation kinetics of the N-desethyl metabolite and the parent glucuronides were studies in the same liver preparation from 14 different human livers (HL-Mix 14) to afford a comparison of the oxidation and glucuronidation pathways.

To determine the formation rates of N-desethyl metabolite, AG-024322 with a concentration range of 0.5 $100 \mu \mathrm{M}$, each in triplicate, was pre-incubated with HLM containing $0.75 \mathrm{mg} / \mathrm{ml}$ protein in $100 \mathrm{mM}$ potassium phosphate buffer ( $\mathrm{pH} \mathrm{7.4)} \mathrm{for} 5$ minutes at $37^{\circ} \mathrm{C}$. The reaction was initiated by the addition of $1 \mathrm{mM}$ NADPH in a final incubation volume of $0.5 \mathrm{ml}$. Reactions were terminated at 15 minutes by the addition of $0.5 \mathrm{ml}$ of acetonitrile and $40 \mu \mathrm{l}$ of $1 \mathrm{ng} / \mu \mathrm{l}$ IS. The remaining part of the procedure was performed as described above for CYP reaction phenotyping screen.

The experiment conditions to determine the formation rates of parent glucuronides were optimized in respect to time and protein concentration. Linear product formation was observed for up to 1 hour and $2 \mathrm{mg} / \mathrm{ml} \mathrm{microsomal}$ protein. The experiments were following the similar procedure as described above using UGT supersomes, except that the incubation mixture contains $1 \mathrm{mg} / \mathrm{ml}$ microsome protein, $1 \mathrm{mM} \mathrm{MgCl}_{2}, 0.05 \mathrm{mg} / \mathrm{mg}$ microsome protein alamethicin, and $2.5 \mathrm{mM}$ UDPGA in either $0.1 \mathrm{M}$ phosphate buffer ( $\mathrm{pH} 7.1$ ) or WEM, incubated with a range of AG-024322 concentrations ( 0.5 to 200 $\mu \mathrm{M})$ in triplicate, and terminated at 35 minutes.

\subsection{Formation Kinetics Calculation}

The relative formation rate of each metabolite (peak area ratio/mg protein/15 or $35 \mathrm{~min}$ ) was calculated and plotted against AG-024322 concentrations. The metabolite formation kinetic parameter, $K_{\mathrm{m}}$, was estimated by fitting the data to the sigmoid Emax model using SAAMII (SAAM Institute, Seattle, WA). The maximum formation rate $\left(V_{\max }\right)$ was estimated from separate experiments of HLM incubation with $10 \mu \mathrm{M}\left[{ }^{14} \mathrm{C}\right] \mathrm{AG}-024322$ at the similar conditions as described above. The formation rate $(V)$ of each metabolite in the incubations was calculated based on the radioactivity. The $V_{\max }$ value was then calculated based on the Michaelist-Menten equation, $V_{\max }$ is the substrate concentration. The in vitro intrinsic clearance $\left(C L_{\text {int }}\right)$ was determined by $V_{\max } / K_{\mathrm{m}}$ ratio. The predicted $C L_{\text {int }}$ was scaled up to in vivo by extrapolating $C L_{\text {int }}$ assuming a human liver weight of $1500 \mathrm{~g} \mathrm{(70} \mathrm{kg} \mathrm{body}$ weight) and that one gram liver tissue contains $45 \mathrm{mg}$ HLM protein. The total hepatic clearance $\left(C L_{\mathrm{h}}\right)$ was calculated 
from the well-stirred model: $C L_{\mathrm{h}}=Q \times C L_{\text {int }} / Q+C L_{\text {int }}$, where $Q$ is the human hepatic blood flow $(20 \mathrm{ml} / \mathrm{min} / \mathrm{kg})$. The estimation of $C L_{\mathrm{h}}$ was not incorporated with plasma and microsome protein binding.

\subsection{Mass Balance Study in Rats}

In this study, $\left[{ }^{14} \mathrm{C}\right] \mathrm{AG}-024322$ in $5 \%$ dextrose in water (pH 4) was administered as a single 30-minute IV infusion to all animals at a target dose level of $10 \mathrm{mg} / \mathrm{kg}$ ( $\sim 50 \mu \mathrm{Ci} / 250 \mathrm{~g}$ body weight). The intact animals in Group 1 (3/gender) were individually housed in metabolism cages for the separate collection of urine and feces over intervals through 120 hours after dosing. The bile duct-cannulated (BDC) rats in Group 3 (2/gender) were individually housed in metabolism cages for the collection of bile over intervals through 48 hours. Blood samples were collected from intact animals in Group 2 (2/gender) before dosing and at $0.25,0.5,1,3,8,24,48$, 72,96 , and 120 hours from the initiation of infusion. Blood samples were collected from intact animals in Group 4 by sacrificing 1 animal/gender at each time point at $0.5,2,4$, and 8 hours from the initiation of infusion. Urine, feces, bile, cage wash, cage wipe, blood, and plasma samples were processed and analyzed for radioactivity. Selected plasma, urine, bile, and fecal samples were used for metabolite identification and profiling.

\subsection{Plasma, Urine, Fecal and Bile Sample Preparation}

Plasma samples collected from Group 4 animals were pooled based on the pooling formula described by Hamilton and coworkers [7] to generate plasma pools (2.0 $\mathrm{mL})$. The pooled plasma samples were precipitated by the addition of 4 volumes of acetonitrile: methanol (3:1, $\mathrm{v} / \mathrm{v}$ ) with $0.2 \%$ formic acid, and then vortexed and centrifuged. Urine samples were pooled on a percent weight basis and centrifuged. The supernatants from the plasma or urine pool extracts were concentrated to approximately $1 \mathrm{ml}$ under $\mathrm{N}_{2}$ before LC/RAM/MS analysis. Selected fecal homogenate from each animal were pooled on a percent weight basis to generate fecal homogenate pools. Each pooled sample was precipitated by the addition of 4 volumes of acetonitrile containing $0.2 \%$ formic acid, vortexed and centrifuged. The pellet was reextracted using the same method, and supernatants were combined for evaporation under $\mathrm{N}_{2}$ to dryness. The residues were reconstituted in $1 \mathrm{ml}$ of 20:80 (v/v) methanol:20 $\mathrm{mM}$ ammonium acetate with $0.1 \%$ formic acid for LC/RAM/MS analysis. Selected bile samples were pooled on a percent weight basis to generate bile pools. After centrifugation, every $100 \mu \mathrm{l}$ of bile sample was diluted with $900 \mu \mathrm{l}$ of 20:80 (v/v) methano 1:20 mM ammonium acetate containing $0.1 \%$ formic acid for LC/RAM/MS analysis. All centrifugation was performed at $15850 \times \mathrm{g}$ for 10 minutes using Brinkman eppendorf 5415c (West- bury, NY). The injection volume for LC/RAM/MS was 300-900 $\mu 1$.

\subsection{Determination of Radioactivity}

The radioactivity in urine, bile, fecal, blood, and plasma samples was determined by liquid scintillation counting (LSC). Triplicate weighed aliquots (approximately 0.02 to $0.1 \mathrm{~g}$ each) of urine, bile, and plasma samples were mixed with scintillation fluid, and analyzed directly by LSC. Triplicate weighed aliquots of fecal homogenate (fecal:water, 20:80, w/w, approximately 0.1-0.5 g each) or blood (approximately 0.02-0.03 g each) were placed into cones and pad, dried, combusted, and then analyzed by LSC.

\subsection{Metabolite Characterization}

Metabolite characterization was performed using an Agilent 1100 HPLC system (Wilmington, DE) coupled in-line with an ARC Model C StopFlow System (AIM Research Company, Newark, DE) and an IN/US Model $3 \beta$-RAM radio-detector (Tampa, FL), and a ThermoFinnigan LCQ-Deca XP ion trap mass spectrometer (San Jose, CA). When MS detection was used, the HPLC effluent was split with approximately $20 \%$ of the flow introduced into the MS via electrospray ionization (ESI) source and $80 \%$ diverted to the $\beta$-RAM detector. The ion-trap ESI-MS was operated in positive ion model with a spray voltage of $4.5 \mathrm{kV}$, sheath gas flow rate of 70 (arbitrary), auxiliary gas flow rate of 20 (arbitrary) and capillary temperature at $200^{\circ} \mathrm{C}$. Laura $3 \mathrm{~V} 3.0$ (IN/US system, Tampa, FL), ARC data system V2.4 (AIM Research Company, Newark, DE) and Xcalibur V1.4 (ThermoFinnigan, San Jose, CA) software were employed to control the $\beta$-RAM detector, the StopFlow system and the LC-MS system for data acquisition and processing. The MS spectra were acquired over a mass range of $\mathrm{m} / \mathrm{z} 100-900$ for all samples. Ion-trap LC-ESI$\mathrm{MS} / \mathrm{MS}$ experiments were performed to generate ion mass spectra for the selected molecular ions representing possible metabolites of AG-024322. AG-024322 metabolites were identified by chromatographic resolution and concurrent radiometric and mass-selective quantification.

Separation of the parent drug and its metabolites was accomplished using a Phenomenex Luna $5 \mu \mathrm{C} 18$ column $(250 \times 4.6 \mathrm{~mm}$, Torrance, CA $)$ or an Agilent Zorbax $5 \mu$ C8 column $(150 \times 4.6 \mathrm{~mm}$, Palo Alto, CA). The mobile phase consisting of a $20 \mathrm{mM}$ ammonium acetate in water ( $\mathrm{pH} 4.0)$ as Solvent A and acetonitrile as Solvent B, and were programmed for each matrix using a linear gradient at a constant flow rate of $1.0 \mathrm{~mL} / \mathrm{min}$ as follows: 1) for hepatocyte and microsome incubations: $0 \mathrm{~min}, 5 \%$ B; 5 min, 5\% B; $30 \mathrm{~min}, 35 \%$ B; $31 \mathrm{~min}, 95 \% \mathrm{~B}$; $35 \mathrm{~min}$, 95\% B; $36 \mathrm{~min}, 5 \% \mathrm{~B}$; and $43 \mathrm{~min}, 5 \% \mathrm{~B} ; 2$ ) for urine, feces and bile samples: $0 \mathrm{~min}, 10 \% \mathrm{~B} ; 10 \mathrm{~min}, 18 \% \mathrm{~B}$; 
$20 \mathrm{~min}, 18 \% \mathrm{~B} ; 42 \mathrm{~min}, 30 \% \mathrm{~B} ; 50 \mathrm{~min}, 30 \% \mathrm{~B}$; $60 \mathrm{~min}$, $55 \% \mathrm{~B}$; $61 \mathrm{~min}, 10 \% \mathrm{~B}$; and $70 \mathrm{~min}, 10 \% \mathrm{~B}$; and 3 ) for plasma samples: $0 \mathrm{~min}, 10 \% \mathrm{~B} ; 50 \mathrm{~min}, 30 \% \mathrm{~B}$; $60 \mathrm{~min}$, $55 \% \mathrm{~B} ; 61 \mathrm{~min}, 10 \% \mathrm{~B}$; and $70 \mathrm{~min}, 10 \% \mathrm{~B}$.

The analysis of $\mathrm{N}$-desethylation metabolite of AG024322 and the glucuronides of the parent drug was using a Zorbax C8 column $(2.1 \times 5 \mathrm{~mm}, 5 \mu)$ from Agilent (Palo Alto, CA) to allow a chromatographic resolution of the metabolites from the parent drug with a run time of 12 minute. With an API 4000 MS (AB Sciex Instruments, Toronto, Ontario), the monitored ion transitions were $419 \rightarrow 374,391 \rightarrow 374$ and $595 \rightarrow 374$ for the parent compound, N-desethyl metabolite and parent glucuronide, respectively. The mobile phase was programmed from the initial setting at $95 \%$ Solvent C (2 $\mathrm{mM}$ ammonium acetate with $0.1 \%$ formic acid in water) and 5\% Solvent D (acetonitrile with $0.1 \%$ formic acid) to $35 \% \mathrm{C}$ and $65 \% \mathrm{D}$ at 10 minutes by linear gradient, and restored to original composition at $10.1 \mathrm{~min}$ until the end of run.. The flow rate was maintained constant at $0.3 \mathrm{ml} / \mathrm{min}$.

\section{RESULTS}

\subsection{In Vitro Metabolism of AG-024322}

Metabolic stability of $\left[{ }^{14} \mathrm{C}\right] \mathrm{AG}-024322$ in liver microsomes and hepatocytes are presented in Table $\mathbf{1}$ as percent of parent drug remaining after incubation in the preparations for rat, dog, monkey and human. The corresponding disappearance half-life was generated from the available time course data. $\left[{ }^{14} \mathrm{C}\right] \mathrm{AG}-024322$ depleted most extensively in rat liver microsome with $11 \%$ of radiochromatogram of the parent drug remaining at 1 -hour incubation time, followed by dog (55\%). Com- parable incubations in human and monkey liver microsomes yielded significantly less extensive parent drug depletions with $95 \%$ and $92 \%$ remaining after 1 -hour incubation, respectively. $\left[{ }^{14} \mathrm{C}\right] \mathrm{AG}-024322$ also exhibited metabolic stability in human hepatocytes relative to other species. After 5 hours incubation, only 14\% of the compound was metabolized in human hepatocytes, whereas $26 \%$ and $27 \%$ in dog and rat hepatocytes, respectively. Monkey hepatocytes most extensively metabolized AG-024322 up to $38 \%$. Based on the chromatographic retention time and molecule weight, several metabolites were tentatively identified as parent glucuronides (M1, M2, and M3), N-desethylation (M6) and metabolites that may arise from $\mathrm{N}$-acetylation of M6 (M8) or parent methylation (M10) in hepatocytes and microsomes of various species (Table 2). The radiochro- matograms of the 5-hour incubations of $\left[{ }^{14} \mathrm{C}\right] \mathrm{AG}-024322$ in rat, dog, monkey and human hepatocytes are shown in Figure 1. At 5 hours, glucuronidation of the parent drug accounted for approximately $8 \%$ of the total radioactivity, while M6 and M8/M10 accounted for $4 \%$ and $2 \%$ of

Table 1. Metabolic stability of $\left[{ }^{14} \mathrm{C}\right] \mathrm{AG}-024322$ in liver microsomes and hepatocytes.

\begin{tabular}{ccccc}
\hline \multirow{2}{*}{ Species } & \multicolumn{2}{c}{ Microsomes $^{a}$} & \multicolumn{2}{c}{ Hepatocytes $^{b}$} \\
\cline { 2 - 5 } & $\begin{array}{c}\text { Remaining } \\
\text { at 1-hour (\%) }\end{array}$ & $\begin{array}{c}\text { Half-life } \\
\text { (h) }\end{array}$ & $\begin{array}{c}\text { Remaining } \\
\text { at 5-hour(\%) }\end{array}$ & $\begin{array}{c}\text { Half-life } \\
\text { (h) }\end{array}$ \\
\hline Human & 95 & 12 & 86 & 50 \\
Monkey & 92 & 37 & 62 & 8.5 \\
Dog & 55 & 1.0 & 74 & 14 \\
Rat & 11 & 0.3 & 73 & 15 \\
\hline
\end{tabular}

a. Incubations contain $0.75 \mathrm{mg} / \mathrm{ml}$ microsomal protein; $b$. Incubations contain $1.2,1.0,1.8$ and $1.5 \times 10^{6}$ cells $/ \mathrm{ml}$ in WEM for human, monkey, dog and rat, respectively.

Table 2. Metabolite profiles of AG-024322 in rat, dog, monkey and human liver microsomes and cryopreserved hepatocytes incubated with $\left[{ }^{14} \mathrm{C}\right] \mathrm{AG}-024322$.

\begin{tabular}{|c|c|c|c|c|c|c|c|c|c|c|}
\hline \multirow{2}{*}{ Species } & \multicolumn{10}{|c|}{$\%$ Radiochromatogram } \\
\hline & M1 & M2 & M3 & M5 (Unk) & M6 & M7(Unk) & AG-024322 & M7/M10 & M9(Unk) & M10/M7 \\
\hline \multicolumn{11}{|c|}{ Liver Microsomes (at 1 Hour of Incubation) } \\
\hline $\mathrm{Rat}^{a}$ & - & - & - & - & 7.5 & 10.6 & 10.8 & 8.8 & 22.1 & - \\
\hline $\operatorname{Dog}^{b}$ & - & - & - & - & 5.2 & 12.3 & 55.3 & 14.0 & - & - \\
\hline Monkey & - & - & - & - & 8.1 & - & 91.9 & - & - & - \\
\hline Human & - & - & - & - & 4.8 & - & 95.2 & - & - & \\
\hline \multicolumn{11}{|c|}{ Hepatocytes (at 5 hours of Incubation) } \\
\hline Rat & - & - & 8.3 & 1.2 & 11.3 & - & 73.1 & 6.1 & - & - \\
\hline Dog & - & - & - & - & 13.6 & - & 74.2 & 2.4 & 3.9 & 5.8 \\
\hline Monkey & 1.9 & 7.3 & 21.7 & 1.8 & 4.5 & 0.8 & 62 & - & - & - \\
\hline Human & 1.5 & 1.5 & 5.2 & - & 4.1 & - & 85.7 & 2.1 & - & - \\
\hline
\end{tabular}

a. Novel unidentified metabolites accounted for $40.2 \%$ of radiochromatogram in rat liver microsome (not listed); b. Novel unidentified metabolites accounted for $13.2 \%$ of radiochromatogram in dog liver microsome (not listed); -: Trace or no RAM detected metabolites; M1, M2 and M3: Parent drug glucuronides (M.W. 594); M6: N-desethyl AG-024322 (M.W. 386); M7/M10: acetylated M6 or methylated AG-024322 (M.W. 432); Unk: unknown/unidentified metabolite. 

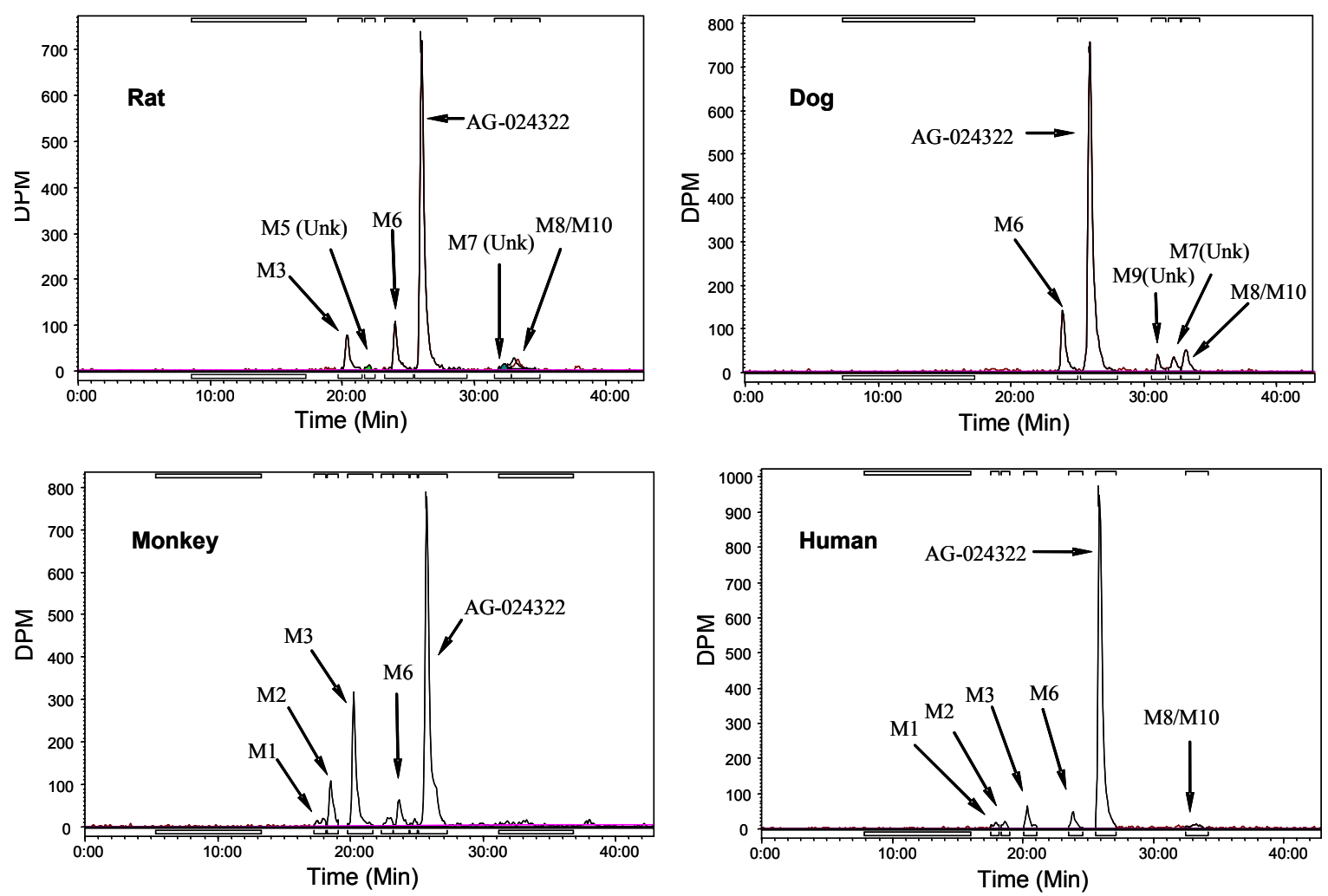

Figure 1. Representative HPLC radiochromatograms of a 5-hour $\left[{ }^{14} \mathrm{C}\right] \mathrm{AG}-024322(11 \mu \mathrm{M})$ incubation in rat, dog, monkey and human cryopreserved hepatocytes $\left(1 \times 10^{6}\right.$ cells $)$

total radioactivity, respectively, in human hepatocytes. M6 is the only metabolite detected in human liver microsomes and accounted for $5 \%$ of the total radioactivity. The preliminary proposed in vitro metabolic scheme is show in Scheme 2.

\subsection{CYP Reaction Phenotyping}

The CYP enzymes involved in N-desethylation of AG024322 were examined by incubations with microsomes from insect cells expressing cDNA (recombinant) human CYPs and by CYP-selective chemical inhibition. CYP3A appear to predominantly catalyzed the N-desethylation of AG-024322 in vitro at 1 and $10 \mu \mathrm{M}$ (Figure 2). Incubations of AG-024322 with recombinant CYP1A2, 2C8 and 2D6, indicated their minor contribution after scale-up of the velocity using either CYP content or relative activity factors [8,9]. Follow-up incubations with CYP-specific inhibitors suggested that only

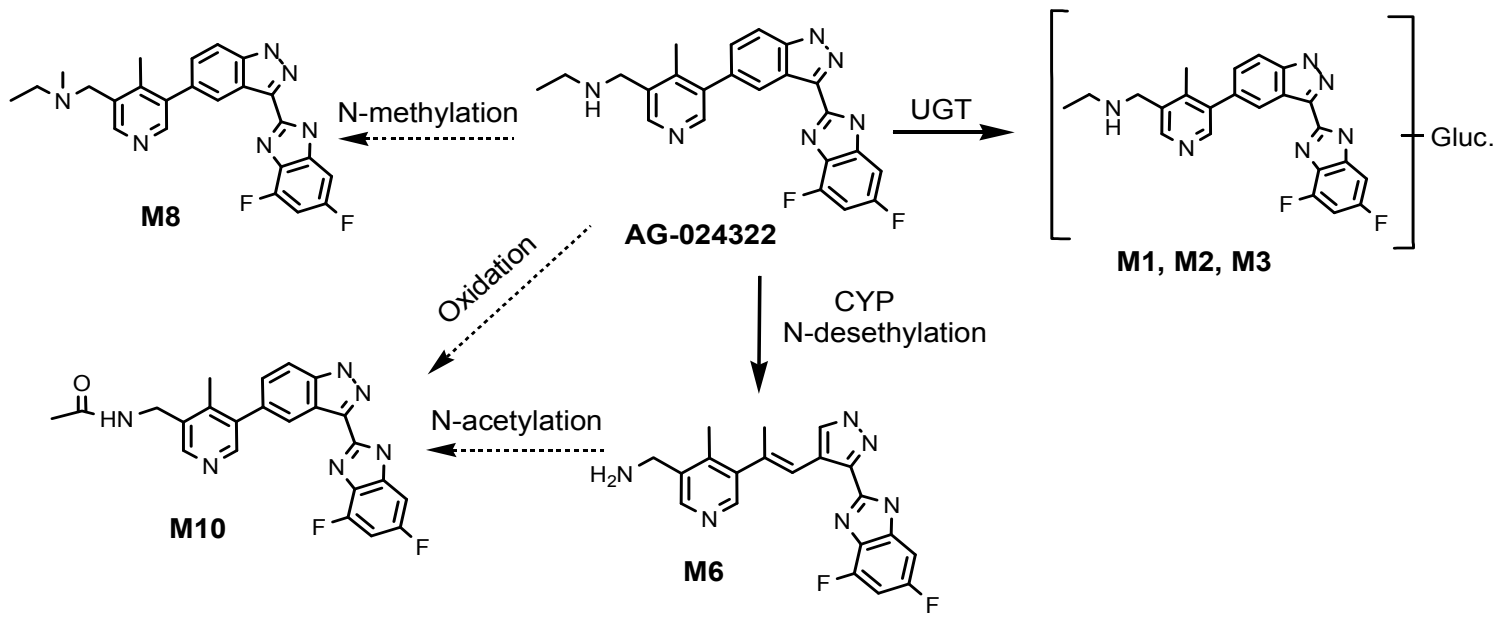

Scheme 2. Preliminary In Vitro AG-024322 metabolic scheme. 
ketoconazole inhibited the N-desethylation of AG-024322 (Table 3). Despite the minor projected involvement of CYP2D6, CYP2C8 and CYP1A2 (Figure 2), quinidine, quercetin and furafyline had no inhibitory effect on the formation rate of $\mathrm{N}$-desethyl AG-024322, confirming a minimal contribution of these enzymes. The involvement of CYP2C9, CYP2C19 and CYP2E1 in N-desethylation of AG-024322 was found to be negligible. Formation kinetics of N-desethyl AG-024322 in human liver microsome were further investigated over the concentration range of 0.5 to $100 \mu \mathrm{M}$ AG-024322 and were best described by a single enzyme system with an apparent $K_{\mathrm{m}}$ of $10 \mu \mathrm{M}$ using SAAMII, a general nonlinear kinetic modeling program (Figure 3). The estimated kinetic parameters, $K_{\mathrm{m}}, V_{\max }$, in vitro $C L_{\mathrm{int}}$, and extrapolated in vivo intrinsic and hepatic clearance are presented in Table 4.

\subsection{UGT Reaction Phenotyping}

The strategy used here for UGT phenotyping is based onwell-established methods, including screening of re

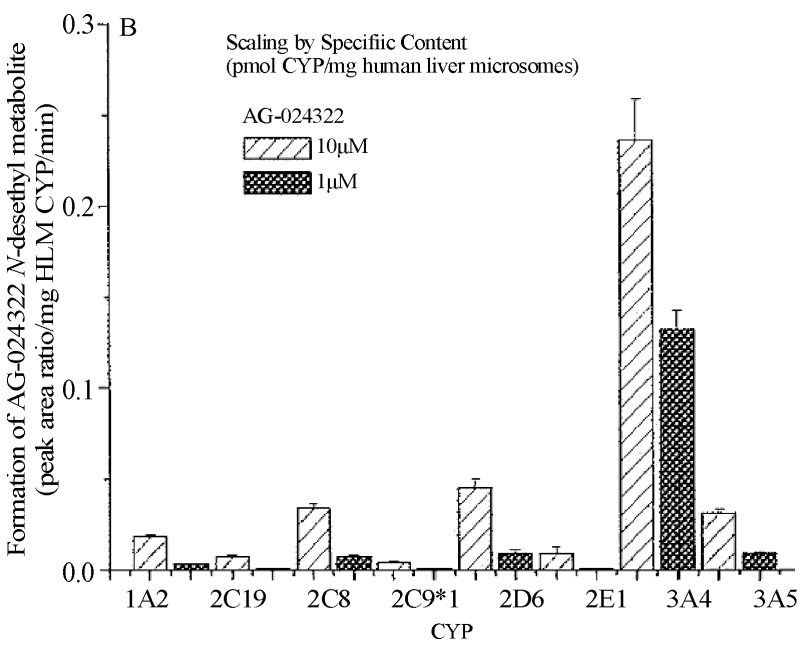

Figure 2. Comparison of AG-024322 N-desethylation activity at 1 and $10 \mu \mathrm{M}$ in microsomes from insect cells individually expressing human CYPs (CYP supersomes).

Table 3. Percent (\%) inhibition or stimulation of AG-024322 metabolism in the presence of CYP-selective Inhibitors.

\begin{tabular}{cccc}
\hline Inhibitor & Concentration $(\mu \mathrm{M})$ & $\begin{array}{c}\text { \% Desethylaton Activity } \\
\text { Remaining at } 1 \mu \mathrm{M} \text { AG-0243222 }\end{array}$ & $\begin{array}{c}\text { \% Desethylaton Activity } \\
\text { Remaining at } 10 \mu \mathrm{M} \text { AG-0243222 }\end{array}$ \\
\hline Ketoconazole & 0.02 & $77.5 \pm 4.4$ & $86.2 \pm 3.9$ \\
(Inhibitor of CYP3A4) & 0.1 & $31.4 \pm 2.2$ & $62.2 \pm 5.2$ \\
& 0.5 & $16.9 \pm 0.5$ & $30.7 \pm 1.7$ \\
Quinidine & 1 & $14.2 \pm 0.4$ & $23.8 \pm 2.1$ \\
(Inhibitor of CYP2D6) & 0.02 & $131 \pm 18$ & $117 \pm 7$ \\
& 0.1 & $157 \pm 11$ & $115 \pm 4$ \\
Quercetin & 0.5 & $158 \pm 8$ & $122 \pm 11$ \\
(Inhibitor of CYP2C8) & 1 & $165 \pm 4$ & $120 \pm 9$ \\
& 0.3 & $145 \pm 6$ & $120 \pm 8$ \\
Furafyline & 2 & $160 \pm 5$ & $120 \pm 9$ \\
(Inhibitor of CYP1A2) & 1 & $156 \pm 3$ & $126 \pm 16$ \\
\hline
\end{tabular}

-: not determined

Table 4. Enzyme kinetic (michalis-menten) parameters estimates of the formation of AG-024322 oxidation and glucuronide metabolites using HL-14 human liver microsome preparation.

\begin{tabular}{|c|c|c|c|c|c|}
\hline Formation Kinetics & Buffer & $K_{\mathrm{m}}(\mu \mathrm{M})$ & $V_{\max }{ }^{a}(\mathrm{pmol} / \mathrm{mg} / \mathrm{min})$ & $C L_{\text {int }}{ }^{b}(\mathrm{ml} / \mathrm{min} / \mathrm{kg})$ & $C L_{h}^{c}(\mathrm{ml} / \mathrm{min} / \mathrm{kg})$ \\
\hline N-desethyl AG-024322 & KPI & 10 & 20 & 1.86 & 1.70 \\
\hline \multirow[t]{2}{*}{ Glucuronide M1 } & KPI & 450 & 35 & 0.07 & 0.07 \\
\hline & WEM & 98 & 77 & 0.74 & 0.72 \\
\hline \multirow[t]{2}{*}{ Glucuronide M2 } & KPI & 9.1 & 5.3 & 0.55 & 0.54 \\
\hline & WEM & 19 & 27 & 1.34 & 1.26 \\
\hline \multirow[t]{2}{*}{ Glucuronide M3 } & KPI & 7.8 & 5.2 & 0.63 & 0.61 \\
\hline & WEM & 11 & 37 & 3.17 & 2.74 \\
\hline
\end{tabular}

a. Formation velocity $V$ was estimated from $\left[{ }^{14} \mathrm{C}\right] \mathrm{AG}-024322$ incubation. $V_{\max }$ was calculated from $V=V_{\max } \times S /\left(K_{\mathrm{m}}+S\right)$, where $S$ is the substrate concentration; b. $C L_{\text {int }}$ was scaled up to in vivo by extrapolating in vitro CLint assuming a human liver weight of $1500 \mathrm{~g}$ (70 kg body weight) and that one gram liver tissue contains $45 \mathrm{mg}$ HLM protein; c. $C L_{h}$ was calculated from the well-stirred model: $C L_{h}=Q \times C L_{\text {int }} / Q+$ $C L_{\text {int }}$, where $Q$ is the human hepatic blood flow $(20 \mathrm{ml} / \mathrm{min} / \mathrm{kg})$; KPI: phosphate buffer, pH 7.1, WEM: William E Medium. 


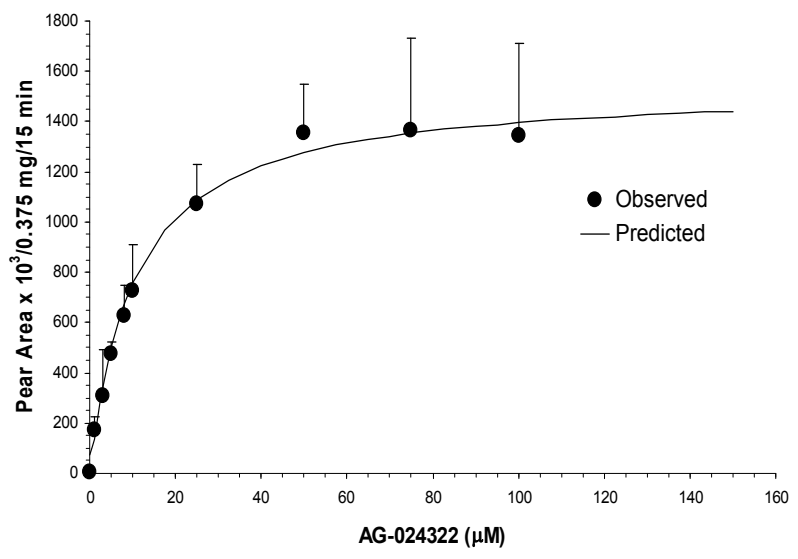

Figure 3. Formation kinetics of N-desethyl AG-024322 in human liver microsomes over the AG-024322 concentration range of 0.5 to $100 \mu \mathrm{M}$.

binant UGTs for activity and comparative enzyme kinetic analysis. Incubations with microsomes from cells individually expressing human UGT1A enzymes (UGT supersomes) yield 3 distinct glucuronide products of the parent drug (M1, M2 and M3), corresponding to the peaks seen after incubation in human hepatocytes. A majority involvement of UGT1A1 at 1 and $10 \mu \mathrm{M}$ of AG-024322 (Figure 4) in the formation of parent drug glucuronides, M2 and M3, and minor involvement of UGT1A8 in the formation of M1 and M3 were observed. The involvement of other UGT1A and UGT2B enzymes in glucuronidation of AG-024322 was negligible. The formation of these three glucuronides was further investigated in HLM incubated at an AG-024322 concentration range of 0.5 to $200 \mu \mathrm{M}$, with the system optimized for glucuronidation. The formation of AG-024322 glucuronides in HLM incubations was also tested in two buffer systems, the phosphate buffer (pH 7.1) and WEM. The data fits and the estimated kinetic parameters for the

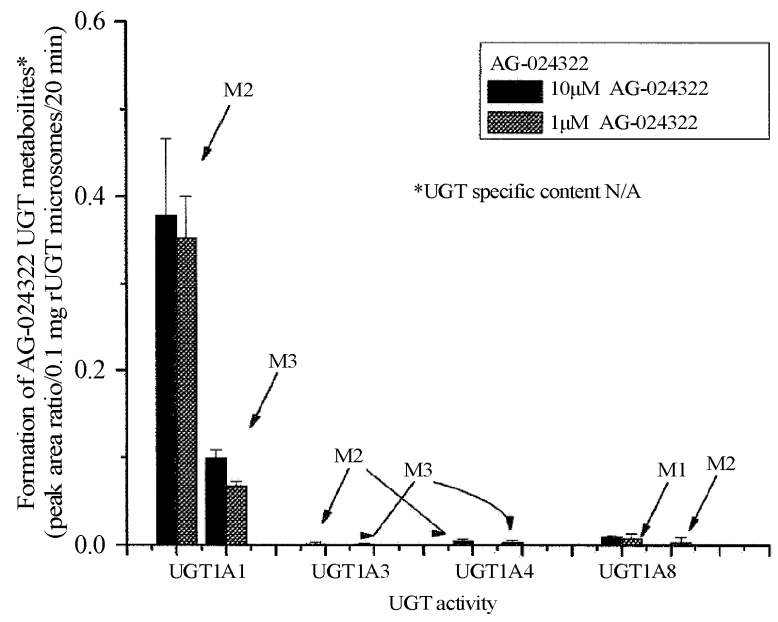

Figure 4. Comparison of AG-024322 glucuronidation (UGT) activity at 1 and $10 \mu \mathrm{M}$ in microsomes from insect cells individually expressing human UGT1A enzymes (UGT supersomes).

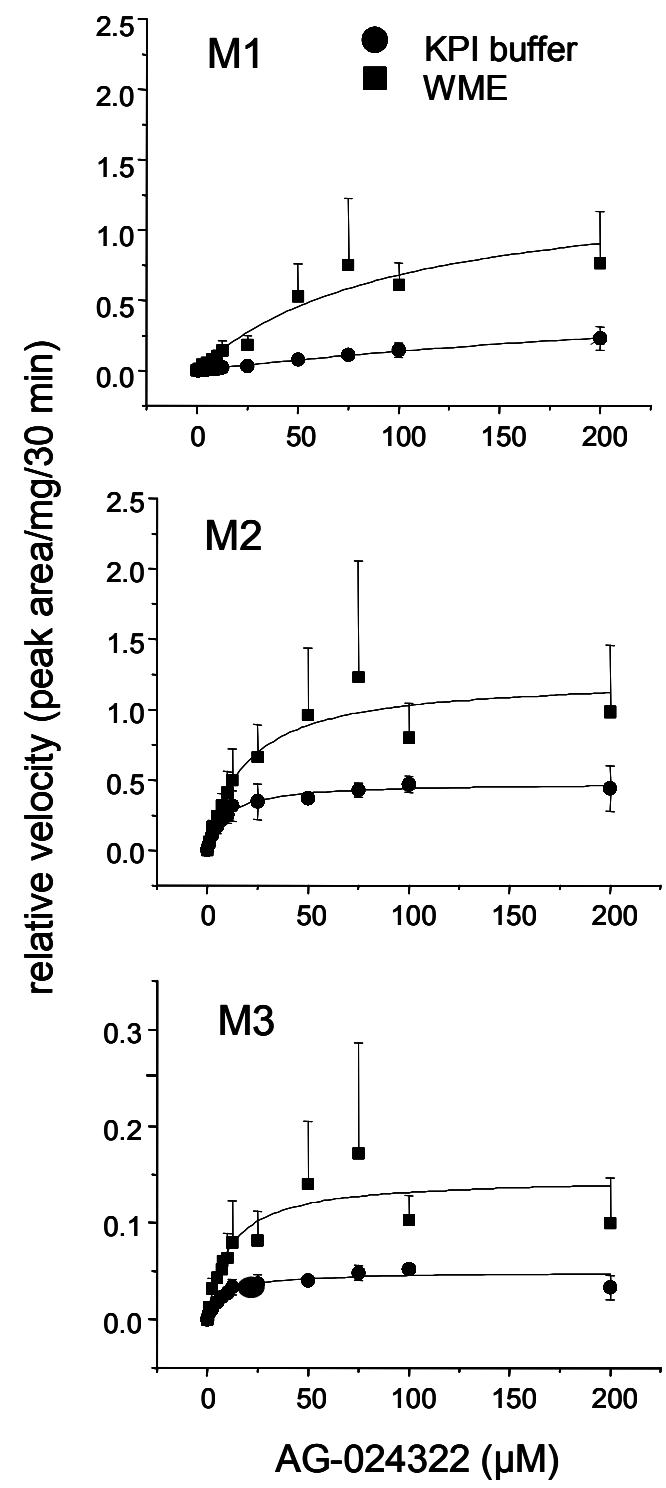

Figure 5. Comparison of formation kinetics of AG024322 glucuronides (M1, M2 and M3) in human liver microsomes (HL-14) using $100 \mathrm{mM}$ phosphate buffer (KPI) or WEM (glucose $16 \mathrm{mM}$ ) in the liver microsome incubation.

glucuronidation pathways are shown in Figure 5 and Table 4, respectively. The extrapolated total in vivo hepatic clearance via parent drug glucuronidation was 1.22 and $4.72 \mathrm{ml} / \mathrm{min} / \mathrm{kg}$ using the phosphate buffer and WEM, respectively.

\section{4. $\left[{ }^{14} \mathrm{C}\right] \mathrm{AG}-024322$ Excretion in Rats}

After a 30-min IV infusion of $\left[{ }^{14} \mathrm{C}\right] \mathrm{AG}-024322$ to intact Sprague-Dawley rats at $10 \mathrm{mg} / \mathrm{kg}(\sim 50 \mu \mathrm{Ci} / 250 \mathrm{~g}$ ani$\mathrm{mal})$, the total recovery of radioactivity dose averaged $84 \%$ by 120 hours, as shown in Table 5. The primary route of excretion of radioactivity was via the feces 
(76.5\%), indicating extensive biliary excretion. A mean total of $56 \%$ of dose was recovered in bile within 48 hours of administration to bile duct-cannulated rats, consisting with the high recovery of radioactivity in feces from the intact rats. Urinary excretion was minimal with an average radioactivity recovery of $3.2 \%$. These data confirmed that the biliary excretion is the major route of elimination in rats, which is consistent with the findings from in vitro metabolism studies. The rate of elimination of radioactivity was moderately rapid and appeared to be essentially complete by 96 hours postdose $(76.1 \%$ in feces and $3.2 \%$ in urine). The overall excretion of radioactivity was similar for males and females.

\subsection{Metabolism of AG-024322 in Rats}

Profiling and identification of metabolites of AG-024322 in rats were conducted for plasma, urine, fecal and bile samples from the excretion mass balance study in rats as described above. AG-024322 underwent extensive metabolism in rats. The representative HPLC-radiochromatograms from rat plasma, urine, feces, and bile are presented in Figure 6. Two major metabolites were identified including a direct glucuronide of the parent drug and M13, a glucuronidation product of M15. M15 is a desethyl aminated metabolite (an alcohol) of AG-024322. Other metabolites identified included the N-desethylated product of AG-024322 (M6), a combined mono-xygenated and dehydrogenated product at the ethyl amine group of AG-024322 (M16), M15, and the glucuronides of M6 and M16 (M12 and M14, respectively). The proposed metabolic scheme of AG-024322 in rats is shown in Scheme 3. The profiles of these metabolites in plasma, urine, feces, and bile from intact rats and bile-duct cannulated rats are presented in Table 6. In plasma, the

Table 5. Recovery (Mean \pm SD) of $\left[{ }^{14} \mathrm{C}\right] \mathrm{AG}-024322$ in Rats Following 30-min IV Infusion of $\left[{ }^{14} \mathrm{C}\right] \mathrm{AG}-024322$ at 10 $\mathrm{mg} / \mathrm{kg}(\sim 50 \mu \mathrm{Ci} / \mathrm{Rat})$.

\begin{tabular}{|c|c|c|c|c|c|}
\hline & \multirow{2}{*}{ Sample } & \multicolumn{4}{|c|}{ Total Radioactivity Recovered (\%) } \\
\hline & & Time Interval & Male $(n=3)$ & Female $(\mathrm{n}=2)^{a}$ & Overall $^{b}$ \\
\hline \multirow[t]{5}{*}{ Intact Rats } & Urine & $0-120 \mathrm{~h}$ & $4.06 \pm 0.24$ & 2.4 & $3.4 \pm 0.94$ \\
\hline & Feces & $0-120 \mathrm{~h}$ & $74.2 \pm 6.7$ & 78.8 & $76.1 \pm 6.3$ \\
\hline & Cage Wash & $0-120 \mathrm{~h}$ & $7.28 \pm 12.3$ & 0.31 & $4.47 \pm 9.52$ \\
\hline & Cage Wipe & $0-120 \mathrm{~h}$ & $0.24 \pm 0.21$ & 0.36 & $0.29 \pm 0.23$ \\
\hline & Total & $0-120 \mathrm{~h}$ & $85.8 \pm 9.4$ & 81.8 & $84.2 \pm 7.7$ \\
\hline Bile Duct-Cannulated Rats & Bile & $0-48 \mathrm{~h}$ & $55.5 \pm 11.2(\mathrm{n}=2)$ & & \\
\hline
\end{tabular}

a. one female rat excluded from calculation of the mean, as a result of difficulties during infusion; $b$. male and female combined.
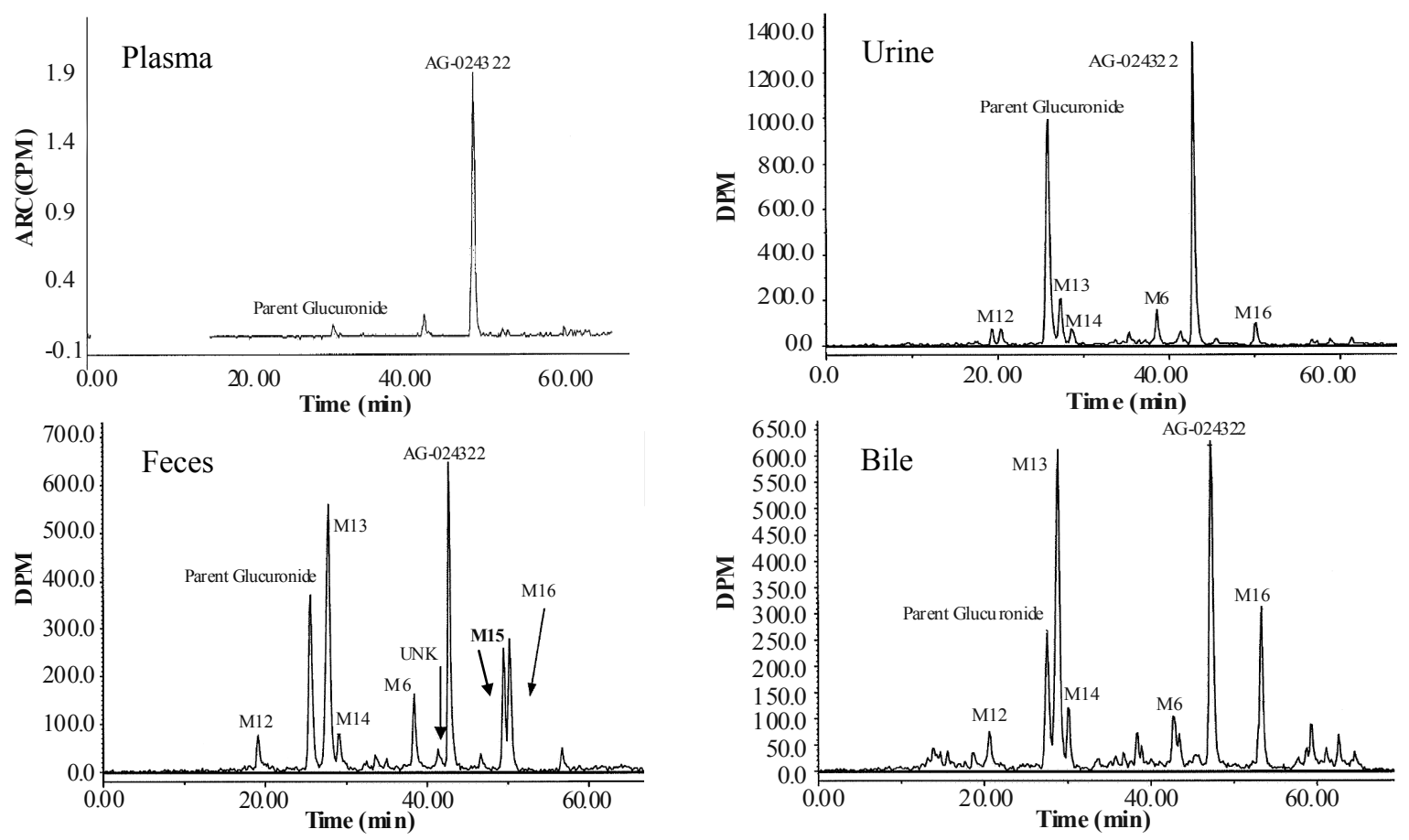

Figure 6. Representative radiochromatograms of plasma, urine, fecal, and bile samples from rats following 30-minutes IV infusion of $\left[{ }^{14} \mathrm{C}\right] \mathrm{AG}-024322$ at $10 \mathrm{mg} / \mathrm{kg}(50 \mu \mathrm{Ci} / 250 \mathrm{~g}$ body weight $)$. 
parent drug was the predominant radioactive component, accounting for $80-88 \%$ of the circulating radioactivity, whereas the parent glucuronide represented $3-5 \%$ of circulating radioactivity. In urine, the parent drug and parent glucuronide represented the two major radioactive components, accounting for $1-1.2 \%$ and $0.6-1.2 \%$ of the dose, respectively. In feces, the major drug-related components included the parent drug, the parent glucuronide and M13, accounting for $9-19 \%, 12-29 \%$ and $9-21 \%$ of the dose, respectively. In bile-duct cannulated male rats, the major biliary drug-related components were the parent drug, the parent glucuronide and M13, accounting for $16 \%, 9 \%$ and $8 \%$ of the dose, respectively.

\subsection{Structure Elucidation of In Vivo Metabolites in Rats}

The tentative structures of the metabolites observed in rats were proposed based on the product ion mass spectrum generated for each identified metabolite peak by ionspray LC-MS/MS. The combined Q1, product ion, and multiple reaction monitoring scanning techniques were used for the structure elucidation. The proposed structure of each metabolite, along with the interpretation of its MS/MS spectrum is presented in Scheme 3. The parent glucuronide had an radiochromatogram retention time of $25.4 \mathrm{~min}$ and an $[\mathrm{M}+\mathrm{H}]^{+}$ion at $\mathrm{m} / \mathrm{z} 595$ $(=419+176)$ consistent with a direct glucuronide of the parent AG-024322. The product ion mass spectrum showed major fragment ions at 550, 419, and 374. M6 had a retention time of $38.2 \mathrm{~min}$ and showed an $[\mathrm{M}+\mathrm{H}]^{+}$ ion at $\mathrm{m} / \mathrm{z} 391(=419-28)$ resulted from N-desethylation of AG-024322. The product ion mass spectrum of M6 gave a major fragment ion of 374 . M12 had a retention time of $19.2 \mathrm{~min}$ and an $[\mathrm{M}+\mathrm{H}]^{+}$ion at $\mathrm{m} / \mathrm{z} 567(=419$ $28+176$ ) consistent with a glucuronide of M6. The product ion mass spectrum of M12 gave fragment ions of 550, 391 and 374. M15 showed a retention time of $49.2 \mathrm{~min}$, and exhibited $[\mathrm{M}+\mathrm{H}]^{+}$at $\mathrm{m} / \mathrm{z} 392(=419-27)$, which is likely formed through desethyl amination of AG-024322. The product ion mass spectra gave fragment ions of 392 and 374. M13 exhibited $[\mathrm{M}+\mathrm{H}]^{+}$at $\mathrm{m} / \mathrm{z} 568(=419-27+176)$ consistent with a glucuronide of M15. The retention time of M13 was $27.0 \mathrm{~min}$ and the MS/MS mass spectrum yield fragment ions of 568 and 392. M16 exhibited $[\mathrm{M}+\mathrm{H}]^{+}$at $\mathrm{m} / \mathrm{z} 433(=419+14)$, re-

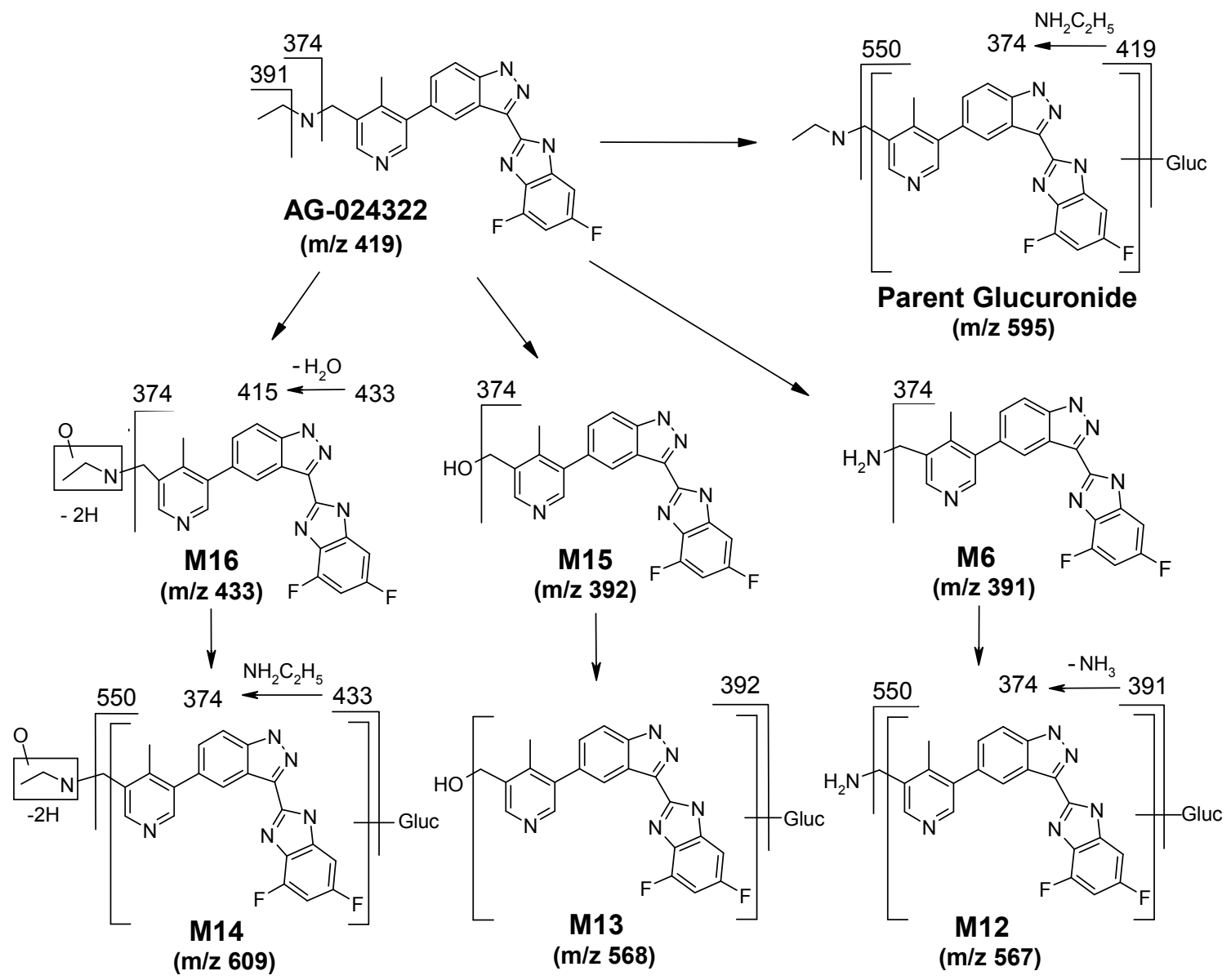

Scheme 3. Proposed metabolic scheme of AG-024322 in rats. 
Table 6. Metabolite Profiles of AG-024322 in plasma, urine, feces, and bile from rats administered $\left[{ }^{14} \mathrm{C}\right]$ AG-024322 intravenously.

\begin{tabular}{ccccccccccc}
\hline \multirow{2}{*}{$\begin{array}{c}\text { Matrix } \\
\text { (Time) }\end{array}$} & \multirow{2}{*}{ Gender } & \multicolumn{3}{c}{ Mean \% Radiochromatogram for Plasma and Mean \% Dose for Urine, Feces and Bile } \\
\cline { 3 - 11 } & & Parent Gluc & M6 & M12 & M13 & M14 & Unk & AG-024322 & M15 & M16 \\
\hline Plasma & Pooled male & 4.52 & $\times$ & $\times$ & $\times$ & $\times$ & $\times$ & 87.70 & $\times$ & $\checkmark$ \\
& Pooled female & 2.61 & $\times$ & $\times$ & $\times$ & $\times$ & $\times$ & 80.06 & $\times$ & $\checkmark$ \\
\multirow{2}{*}{ Urine } & Male average & 1.19 & 0.19 & 0.09 & 0.35 & 0.09 & $\times$ & 1.21 & & 0.13 \\
& Female average & 0.55 & 0.05 & & 0.05 & $\times$ & $\times$ & 1.07 & & $\times$ \\
\multirow{2}{*}{ Feces } & Male average & 11.61 & 2.80 & 2.04 & 21.34 & 3.16 & 1.43 & 9.44 & 3.16 & 4.36 \\
& Female average & 28.98 & 1.04 & 1.66 & 9.39 & 1.20 & 2.97 & 18.97 & 1.86 & 1.15 \\
\multirow{2}{*}{ Bile } & Male average & 8.99 & 2.62 & 1.14 & 8.28 & 1.19 & $\times$ & 16.13 & $\times$ & 4.13 \\
\hline
\end{tabular}

$\times$ : not detectable using either LC-MS or RAM; $\checkmark$ : Trace metabolites detected using LC-MS only; Parent Gluc: parent glucuronide, M1, M2 or M3 (M.W. 594); M6: N-desethylated metabolite of AG-024322 (M.W. 386); M12: Glucuronide of M6 (M.W. 566); M13: Glucuronide of M15 (M.W. 567); M14: Glucuronide of M16 (M.W. 608); M15: Desethyl amination metabolite of AG-024322 (M.W. 391 ); M16: Combined mono-oxygenation and dehydrogenation product of AG-024322 (M.W. 432); Unk: Unknown/unidentified metabolite.

sulted from a combined mono-oxygenation and dehydrogenation of AG-024322. The retention time of M16 was $50 \mathrm{~min}$, and its MS/MS mass spectra gave fragment ions at 433,415 and 374 . M14 demonstrated $[\mathrm{M}+\mathrm{H}]^{+}$at $\mathrm{m} / \mathrm{z} 609(=419+14+176)$ consistent with a glucuronide of M16. M14 had a retention time of $28.3 \mathrm{~min}$ and its mass spectra gave major fragment ions of 609, 550, 433 and 374. The chemical structure of unknown (Unk) cannot be determined with no mass spectrum data available.

\section{DISCUSSIONS}

The in vitro metabolism of AG-024322 was initially investigated in liver microsomes incubated with $\left[{ }^{14} \mathrm{C}\right] \mathrm{AG}-$ 024322 to assess the metabolic stability across species. The experiments were performed with NADPH only to reflect Phase 1 metabolic activity. Subsequent studies were also conducted using hepatocytes, which has a complete ensemble of metabolizing enzymes of all isoforms, cofactors, and cellular components, providing an alternate assessment of in vivo clearance particularly for compounds that are mainly cleared via glucuronidation $[10,11]$. AG-024322 exhibited the highest metabolic stability in both human hepatocytes and liver microsomes as compared to other nonclinical species (Table 1). Extensive microsomal metabolism in the rat and dog was observed, which is consistent with the high in vivo clearance observed in these species. The metabolites identified in human liver microsomes and hepatocytes were also observed in the nonclinical species evaluated. Several in vitro metabolites of AG- 024322 were tentatively identified from microsome and hepatocyte incubations using chromatographic resolution, and concurrent radiometric and mass-selective quantification (Figure 1; Scheme 2). These metabolites including the unidentified metabolites as well as the parent drug account for all the major peaks of radioactivity in microsomes and hepatocytes. The N-desethyl metabolite and glucuronides of the parent drug were major metabolites observed across species. The monkey exhibited the highest extent of glucuronidation in hepatocytes, while dog and rat demonstrated more extensive N-desethylation and methylation/ $\mathrm{N}$-acetylation in hepatocytes, and produced novel unidentified metabolites in microsomes. Metabolism of AG024322 in human hepatocytes and HLM is more comparable to those in monkeys. Both species demonstrated glucuronodation and oxidative $\mathrm{N}$-desethylation as the predominant metabolic pathways.

The excretion mass balance study conducted in rats demonstrated that the primary route of excretion of $\left[{ }^{14} \mathrm{C}\right] \mathrm{AG}-$ 024322 was via feces $(76.5 \%)$ with minimal contributions $(3.2 \%)$ of urinary excretion (Table 5). The high fecal elimination is reflected by biliary elimination that accounted for approximately $56 \%$ of the total radioactivity within 48 hours of dosing. As proposed in Scheme 3, the glucuronide of the parent drug and M6 identified in vitro were also observed in rats following 30-minute IV infusion of $\left[{ }^{14} \mathrm{C}\right] \mathrm{AG}-024322$. Only one parent glucuronide, likely M3 based on the retention time, was observed in rats, which was consistent with the findings that only M3 was detected in the rat hepatocyte incubations (Figure 1 and Table 2). The parent glucuronide appeared to be the most abundant metabolite in plasma and in all excreta collected, representing up to $29 \%$ of dose in feces. Another major metabolite, M13, was the glucuronidation product of a desethyl aminated metabolite (M15), representing up to $21 \%$ of dose in feces, as UGTs are primarily involved in conjugation of metabolites from oxidation reactions. M6, M15 and M16 were oxidative metabolites, each representing less than $5 \%$ of dose, and were also further conjugated to its glucuronides. The metabolite profile in bile (Table 6) is supportive to the findings of extensive biliary excretion, which involves conjugation of the parent drug and oxidative metabolites with glucuronic acid prior to excretion into the bile and direct secretion of the parent drug into bile. 
Reaction phenotyping studies using the standard battery of microsomes from insect cells expressing individual human CYPs or UGTs were conducted to assess the two principal pathways of AG-024322 biotransformation; oxidation and glucuronidation. The formation of M6 in human recombinant demonstrated the predominant role of CYP3A4 enzyme in the oxidative metabolism of AG024322. The relative contribution of the identified CYPs was subsequently examined by inhibition studies with CYP-selective inhibitors. As shown in Table 2, only ketoconazole (selective CYP 3A inhibitor) inhibited Ndesethylation of AG-024322 with up to $86 \%$ inhibition in HLM over an 8-minute of incubation. Quinidine, quercetin or furafyline had no inhibitory effects in the formation rate of $\mathrm{N}$-desethylation. Quinidine is also a known CYP3A heterotropic activator [12], and in this case yields indirect additional evidence towards predominant participation of CYP3A enzymes (Table 3). Formation kinetics of this pathway were also examined in a set of representative human liver microsomes and resulted in an apparent $K_{\mathrm{m}}$ of $10 \mu \mathrm{M}$ and $V_{\max }$ of 20 $\mathrm{pmol} / \mathrm{mg} / \mathrm{min}$. The in vivo $C L_{\mathrm{int}}$ and $C L_{\mathrm{h}}$ extrapolated from the in vitro kinetic data [13] were estimated to be 1.86 and $1.7 \mathrm{ml} / \mathrm{min} / \mathrm{kg}$, respectively. According to the projected human clearance from interspecies scaling (12.8 $\mathrm{ml} / \mathrm{min} / \mathrm{kg}$, data not shown), the formation of M6 was estimated to contribute $13 \%$ of the hepatic clearance.

Glucuronidation catalyzed by UGTs is one of major pathways for drug metabolism and elimination in humans. Identification of the UGTs responsible for glucuronidation of AG-024322 in vitro would assist in the prediction of adverse reactions resulting from drug-drug interactions or genetic polymorphism. The integrated approaches used here for UGT reaction phenotyping were using recombinant enzymes and human liver microsomes with primary focusing on identification of the well-characterized hepatic UGTs, 1A1, 1A3, 1A4, 1A6, 1A8, 1A10, 2B7 and 2B15. The involvement of UGT1 A1 was demonstrated from the UGT recombinant incubation experiments, resulting in three glucuronides, which were identical with the M1, M2 and M3 peaks that also observed in human hepatocyte incubations. UGT1A1 is a polymorphic isoform responsible for the glucuronidation of structurally diverse drugs, non-drug xenobiotics and endogenous compounds (e.g. bilirubin and estrogens) $[14,15]$. It is the primary UGT responsible for bilirubin glucuronidation. UGT1A1 is highly expressed in liver, gastrointestinal tract, and bladder [16-18]. It has been reported that genetic polymorphisms of the UGT1A1 gene were significantly related to severe toxicity of irinotecan [19-21]. AG-024322 showed high affinity towards UGT1A1 in vitro. Potential interaction liability of AG-024322 with drugs metabolized by UGT1 A1 is also an area of great interest and warrants additional studies. It is of concern if glucuronidation via UGT1A1 plays a major role in the elimination of AG-
024322 in humans as competitive inhibition of UGT1A1 may be associated with toxicity, and in patients exhibiting compromised bilirubin metabolism [22].

Formation kinetics of the three glucuronides of AG024322 were further investigated in the same set of human liver preparation as that for the formation kinetic study of oxidative metabolism. Both phosphate buffer ( $\mathrm{pH} 7.1)$ and WEM for this system were evaluated. WEM is a carbonate-based hepatocyte media reagent. Engtrakul [11] examined the effects of microsome incubation condition on the formation of AZT-glucuronide, including various physiological and nonphysiological buffer systems, such as Tris, phosphate, acetate, carbonate, citrate buffers and WEM. Their experiments suggested that both carbonate buffer and WEM produced significantly higher amounts of AZT-glucuronide than other buffers. Therefore, we also included the WEM in the formation kinetic system to compare with the commonly used phosphate buffer. Based on the in vitro $C L_{\text {int }}$, the extrapolated total in vivo $C L_{\text {int }}$ for the three glucuronides, M1, M2 and M3, was 1.25 and $5.25 \mathrm{ml} /$ $\mathrm{min} / \mathrm{kg}$ using phosphate buffer and WEM, respectively, contributing $10 \%$ and $37 \%$ of the predicted human plasma clearance. In agree with the findings by Engtrakul et al. [11], we also found that in the case of glucuronidation of AG-024322, WEM appeared to be a more physiologically relevant buffer yielded almost 4fold higher intrinsic clearance by glucuronidation than the phosphate buffer. Additional studies to examine the impact of WEM, and more importantly, its components to probe the function of UGTs in vitro are warranted. In combination of both contributions for CYP3A mediated $\mathrm{N}$-desethylation and UGT1A1-mediated glucuronidation, total metabolic clearance could account at best for $50 \%$ of the predicted human plasma clearance using WEM. This value may be an underestimation since our calculation did not account for the biliary excretion, formation of oxidative metabolite other than M6, and glucuronidation of Phase I metabolites, which might be significant consideration based on the findings from the excretion study in rats. Caution should be made when extrapolating metabolic lability via glucuronidation of UGT enzymes from HLM, since this system appeared to underestimate the degree of clearance (UGT 1A1 is expressed in human and rat and in general exhibited comparable substrate specificities and efficiencies $\left(V_{\max } / K_{\mathrm{m}}\right)$ of the glucuronide formation, and thus rat and human UGT1A1 are functionally similar $[23,24]$. It is expected to have comparable glucuronidation profiles between rats and humans in vivo.

In our calculation for the well-stirred model, protein binding value was not used. AG-024322 was highly bound to human plasma $(95 \%)$. If the plasma protein binding of AG-024322 is included in the expression describing the well-stirred model of hepatic extraction and if the scaling-up in vitro intrinsic clearance used without 
microsome binding data, the projected in vivo clearance is drastically lower. Obach [25] examined the in vivo hepatic clearance predicted from the in vitro system with the measured in vivo clearance and was found that in some cases, the clearance predicted from in vitro using HLM were substantially lower than those observed in vivo. The compounds exhibiting such a discrepancy were all characterized by high protein binding. When plasma protein binding values were removed from the wellstirred model for these compounds, scaled-up values for clearance were remarkably close to those measured in vivo [26]. Therefore, as an initial estimation of the contribution of the two major metabolic pathways in AG024322 clearance, the protein binding value was not used in the well-stirred model, and was found to be more reasonable in the clearance projection.

\section{CONCLUSIONS}

Investigation of in vitro and in vivo metabolism of AG-024322 demonstrates that UGT1A1 mediated glucuronidation and CYP3A mediated oxidation represent the major metabolic pathways of AG-024322. The estimated contribution of the glucuronidation to total plasma clearance in humans was approximately 3 -fold higher than that from desethylation of AG-024322. Using the WE M buffer system in human liver microsomes to assess the metabolic pathway by glucuronidation improved the clearance predictability of AG-024322. Because of the polymorphism of UGT1A1 and the reported toxicity associated with competitive inhibition of UGT1A1 either by a co-administered drug or in patients exhibiting compromised bilirubin metabolism, this information was used to influence the clinical study design to only include subjects having normal expression of UGT1A1 in the first human study thereby decreasing the toxicity risk to patients.

\section{ACKNOWLEDGMENTS}

Authors would like to thanks Drs. Hai-Zhi Bu and Deepak Dalvie for helpful discussion in structure elucidation of AG-024322 metabolites, Drs. Weiwei Tan and Rachel Courtney for the discussion of the human study design and sharing the human pharmacokinetic data with us, and Drs. Scott Fountain, Bill Smith, Ellen Wu and Bhasker Shetty for their invaluable comments and support of this project.

\section{ABBREVIATIONS}

CDK, cyclin-dependent kinase; HLM, human liver microsome; CYP, cytochrome P450; UGT, UDP-glucurono syltransferase; UDPGA, UDP-glucuronic acid; WEM, Williams E medium; HPLC, high-performance liquid chromatography; MS, mass spectrometry; min, minute(s); IS, internal standard; RAM, radiochemical activ- ity monitor.

\section{REFERENCES}

[1] Zhang, C.C., Kephart, S., McAlpine, I., Nonomiya, J., Higgins, J., Arango, M.E., Yan, Z., Knighton, D., Ferre, R.A., Tikhe, J., et al. (2005) AG-024322 is a potent and selective multi-targeted CDK inhibitor with broad spectrum anti-proliferative activity. Proc Amer Assoc Cancer Res, 46, Abstract \#4415.

[2] Morgan, D.O., Fisher, R.P., Espinoza, F.H., Farrell, A., Nourse, J., Chamberlin, H. and Jin, P. (1998) Control of eukaryotic cell cycle progreesion by phosphorylation of cyclin-dependent kinases. Cancer J Sci Am, 4, S77-83.

[3] Morgan, D.O. (1997) Cyclin-dependent kinases: engines, clocks, and micoprosessors. Annu Rev Cell Biol, 13, 261-291.

[4] Moriarty, K.J., Koblish, H., Johnson, D.L., Galemmo, J. and Robert, A. (2007) Progress in the development of agents. Top Med Chem, 1, 207-291.

[5] Zhang, C.C., Troche, G., Yen, Z., Arango, M.E., Higgins, J., Romero, D., Kephart, S., McAlpine, I., Koudriakova, T. and J. Skaptason, et al. (2005) AG-024322 is a multitargeted CDK inhibitor with potent antitumor activity in vivo. Proc Amer Assoc Cancer Res, 46, Abstract \#4413.

[6] Fisher, M.B., Campanale, K., Ackermann, B.L., VandenBranden, M. and Wrighton, S.A. (2000) In vitro glucuronidation using human liver microsomes and the pore-forming peptide alamethicin. Drug Metab Dispos, 28, 560-566.

[7] Hamilton, R.A., Garnett, W.R. and Kline, B.J. (1981) Determination of mean valproic acid serum level by assay of a single pooled sample. Clin Pharmacol Ther, 29, 409-413.

[8] Venkatakrishnan, K., von Moltke, L.L., Court, M.H., Harmatz, J.S., Crespi, C.L. and Greenblatt, D.J. (2000) Comparison between cytochrome P450 (CYP) content and relative activity approaches to scaling from DNAexpressed CYPs to human liver microsomes: Ratios of accessory proteins as sources of discrepancies between the approaches. Drug Metab Dispos, 28, 1493-1504.

[9] Stormer, E., von Moltke L.L. and Greenblatt, D.J. (2000) Scaling drug biotransformation data from cDNA-expressed cytochrome P-450 to human liver: A comparison of relative activity factors and human liver abundance in studies of mirtazapin metabolism. $J$ Pharmacol Exp Ther, 295, 793-801.

[10] Gomez-Lechon, M.J., Donato, M.T., Castell J.V. and Jover, R. (2004) Human hepatocytes in primary culture: the choice to investigate drug metabolism in man. Curr Drug Metab, 5, 443-462.

[11] Engtrakul, J.J., Foti, R.S., Strelevitz, T.J. and Fisher, M.B. (2005) Altered AZT (3'-azido-3'-deoxythymidine) glucuronidation kinetics in liver microsomes as an explanation for underprediction of in vivo clearance: Comparison to hepatocytes and effect of incubation environment. Drug Metab Dispos, 33, 1621-1627.

[12] Tang, W. and Stearns, R.A. (2001) Heterotropic cooperativity of cytochrome P450 3A4 and potential drug-drug interactions. Current Drug Metabolism, 2, 185-198.

[13] Houston, J.B. (2001) Utility of in vitro drug metabolism 
data in predicting in vivo metabolic clearance. BioChem Pharmacol, 47, 1469-1479.

[14] Jansen, P.L., Mulder, G.J., Burchell, B. and Bock, K.W. (1992) New developments in glucuronidation research: Report of a workshop on "glucuronidation, its role in health and disease". Hepatology, 15, 532-544.

[15] Beutler, E. Gelbart, T. and Demina, A. (1998) Racial variability in the UDP-glucuronosyltransferase 1 (UGT1A1) promoter: A balanced polymorphism for regulation of bilirubin metabolism? Proc Natl Acad Sci, U.S.A., 95, 8170-8174.

[16] Strassburg, C.P., Kneip, S. Topp, J., Obermayer-Straub, P., Barut, A., Tukey, R.H. and Manns, M.P. (2000) Polymorphic gene regulation and interindividual variation of UDP-glucuronosyltransferase activity in human small intestine. J Biol Chem, 275, 36164-36171.

[17] Tukey R.H. and Strassburg, C.P. (2000) Human UDPglucuronosyltransferases: Metabolism, expression, and disease. Annu Rev Pharmacol Toxicol, 40, 581-616.

[18] Nakamura, A., Nakajima, M., Yamanaka, H., Fujiwara R. and Yokoi, T. (2008) Expression of UGT1A and UGT2B mRNA in human normal tissues and various cell lines. Drug Metab Dispos, 60, 1461-1464.

[19] Ando, Y., Saka, H., Ando, M., Sawa, T., Muro, K., Ueoka, H., Yokoyama, A., Saitoh, S., Shimokata, K. and Hasegawa, Y. (2000) Polymorphisms of UDP- glucuronosyltransferase gene and irinotecan toxicity: A pharmacogenetic analysis, Cancer Res, 60, 6921-6926.

[20] Mani, S. (2001) UGT1A1 polymorphism predicts irinotecan toxicity: Evolving proof. AAPS PharmSci, 3, 1208-1303.
[21] Lyer, L., Hall, D., Das, S., Mortell, M.A., Ramirez, J., Kim, S., Di Rienzo, A. and Ratain, M.J. (1999) Phenotype-genotype correlation of in vitro SN-38 (active metabolite of irinotecan) and bilirubin glucuronidation in human liver tissue with UGT1A1 promoter polymorphism. Clin Pharmacol Ther, 65, 576-582.

[22] Kadakol, A., Ghosh, S.S., Sappal, B.S., Sharma, G., Chowdhury, J.R. and Chowdhury, N.R. (2000) Genetic lesions of bilirubin urdine-diphosphoglucuronate glucuronosyltrabsferase (UGT1A1) causing Crigler-Najjar and Gilbert syndromes: correlation of genotype to phenotype. Hum Mutat, 16, 297-306.

[23] King, C.D., Green, M.D., Rios, G.R., Coffman, B.L., Owens, I.S., Bishop, W.P. and Tephly, T.R. (1996) The glucuronidation of exogenous and endogenous compounds by stably expressed rat and human UDP-glucuronosyltrasferase 1.1. Arch Biochem Biophys, 332, 92-100.

[24] King, C.D., Rios, G.R., Green, M.D. and Tephly, T.R. (2000) UDP-glucuronosyltrasferase. Curr Drug Metab, 1, 143-161.

[25] Obach, R.S. (1996) The importance of nonspecific binding in in vitro matrices, its impact on enzyme kinetic studies of drug metabolism reactions, and implications for in vitro-in vivo correlations. Drug Metab Dispos, 24, 1047-1049.

[26] Obach, R.S. (1997) Nonspecific binding to microsomes: Impact on scale-up of in Vitro Intrinsic clearance to hepatic clearance as assessed through examination of warfarin, imipramine, and propranolol. Drug Metab Dispos, 25, 1359-1369. 